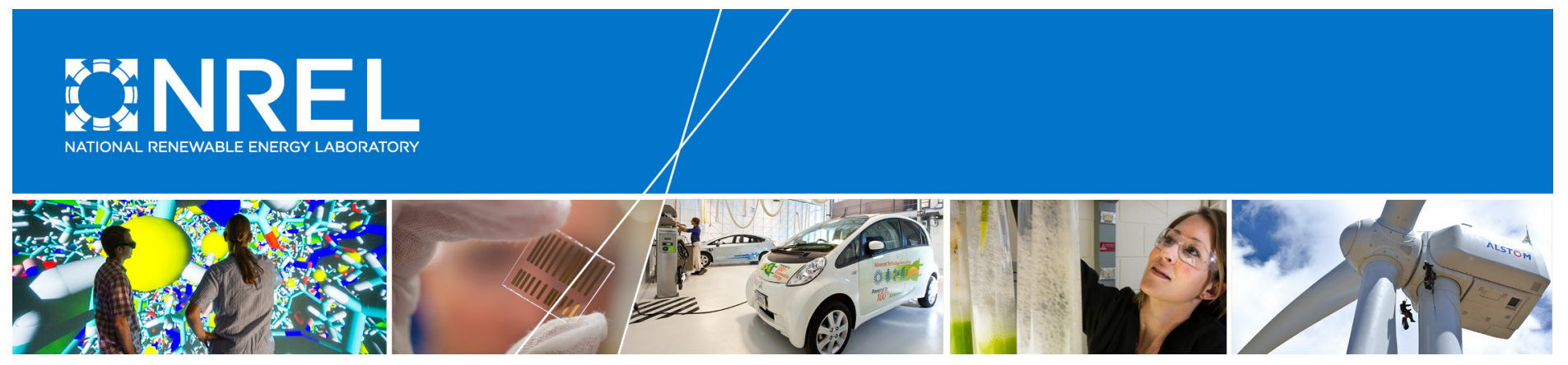

\title{
On the Effects of Wind Turbine Wake Skew Caused by Wind Veer
}

\section{Preprint}

Matthew Churchfield and Senu Sirnivas National Renewable Energy Laboratory

Presented at the American Institute of Aeronautics and Astronautics SciTech

Kissimmee, Florida

January 8-12, 2018

\section{Suggested Citation}

Churchfield, Matt and Senu Sirnivas. 2018. "On the Effects of Wind Turbine Wake Skew Caused by Wind Veer: Preprint." Golden, CO: National Renewable Energy Laboratory. NREL/CP-5000-70686.

www.nrel.gov/docs/fy180sti/70686.

NREL is a national laboratory of the U.S. Department of Energy Office of Energy Efficiency \& Renewable Energy Operated by the Alliance for Sustainable Energy, LLC

This report is available at no cost from the National Renewable Energy Laboratory (NREL) at www.nrel.gov/publications.

\section{Conference Paper}

NREL/CP-5000-70686

March 2018 


\section{NOTICE}

The submitted manuscript has been offered by an employee of the Alliance for Sustainable Energy, LLC (Alliance), a contractor of the US Government under Contract No. DE-AC36-08GO28308. Accordingly, the US Government and Alliance retain a nonexclusive royalty-free license to publish or reproduce the published form of this contribution, or allow others to do so, for US Government purposes.

This report was prepared as an account of work sponsored by an agency of the United States government. Neither the United States government nor any agency thereof, nor any of their employees, makes any warranty, express or implied, or assumes any legal liability or responsibility for the accuracy, completeness, or usefulness of any information, apparatus, product, or process disclosed, or represents that its use would not infringe privately owned rights. Reference herein to any specific commercial product, process, or service by trade name, trademark, manufacturer, or otherwise does not necessarily constitute or imply its endorsement, recommendation, or favoring by the United States government or any agency thereof. The views and opinions of authors expressed herein do not necessarily state or reflect those of the United States government or any agency thereof.

This report is available at no cost from the National Renewable Energy Laboratory (NREL) at www.nrel.gov/publications.

Available electronically at SciTech Connect http:/www.osti.gov/scitech

Available for a processing fee to U.S. Department of Energy and its contractors, in paper, from:

U.S. Department of Energy

Office of Scientific and Technical Information

P.O. Box 62

Oak Ridge, TN 37831-0062

OSTI http://www.osti.gov

Phone: 865.576.8401

Fax: 865.576.5728

Email: reports@osti.gov

Available for sale to the public, in paper, from:

U.S. Department of Commerce

National Technical Information Service

5301 Shawnee Road

Alexandria, VA 22312

NTIS http://www.ntis.gov

Phone: 800.553 .6847 or 703.605 .6000

Fax: 703.605 .6900

Email: orders@ntis.gov 


\title{
On the Effects of Wind Turbine Wake Skew Caused by Wind Veer
}

\author{
Matthew J. Churchfield* and Senu Sirnivas ${ }^{\dagger}$ \\ National Renewable Energy Laboratory, Golden, Colorado, 80401, USA
}

\begin{abstract}
Because of Coriolis forces caused by the Earth's rotation, the structure of the atmospheric boundary layer often contains a wind-direction change with height, also known as wind-direction veer. Under low-turbulence conditions, such as a stably stratified atmosphere, this veer can be significant across the vertical extent of a wind turbine's rotor disk. The veer then causes the wind turbine wake to skew as it advects downstream. This wake skew has been observed both experimentally and numerically. In this work, we attempt to examine the wake skewing process in some detail, and quantify how differently a skewed wake versus a nonskewed wake affects a downstream turbine. We do this by performing atmospheric large-eddy simulations to create turbulent inflow winds with and without veer. In the veer case, there is a roughly $8^{\circ}$ wind-direction change across the turbine rotor. We then perform subsequent large-eddy simulations using these inflow data with an actuator line rotor model to create wakes. The turbine modeled is a large, modern, offshore, multimegawatt turbine. We examine the unsteady wake data in detail and show that the skewed wake recovers faster than the nonskewed wake. We also show that the wake deficit does not skew to the same degree that a passive tracer would if subject to veered inflow. Lastly, we use the wake data to place a hypothetical turbine 9 rotor diameters downstream by running aeroelastic simulations with the simulated wake data. We see differences in power and loads if this downstream turbine is subject to a skewed or nonskewed wake. We feel that the differences observed between the skewed and nonskewed wake are important enough that the skewing effect should be included in engineering wake models.
\end{abstract}

\section{Introduction}

$\mathrm{E}$ NGINEERING wind turbine wake models are important because they are necessary in the estimation of Ewind-power-plant performance. For example, computationally efficient, steady-state wake models like those of Jensen ${ }^{1}$ and Katíc et al. ${ }^{2}$ or Ainslee ${ }^{3}$ are used in wind-power-plant efficiency computations. The computationally efficient, but unsteady, dynamic wake meandering model ${ }^{4}$ can be used for both efficiency computations and mechanical loads predictions.

A commonality to engineering wake models is that they assume axisymmetry of the wake velocity deficit. However, real wind turbine wakes are not necessarily axisymmetric. Certainly they are not axisymmetric instantaneously, and they need not be axisymmetric in the mean. An important situation in which this is true is when there is significant mean inflow wind-direction change vertically across the rotor disk. This variation in mean wind direction with height is known as "wind veer." Wind veer is a direct result of Coriolis effects caused by the Earth's rotation, and more commonly occurs with significant magnitude under stable atmospheric stratification. When a wind turbine is subject to veered inflow winds, as the wake convects downstream, it also skews. In other words, the winds carry the top of the wake downstream and laterally, and they carry the bottom of the wake downstream and to the opposite lateral direction. This effect has been observed in full-scale-turbine wakes with scanning-lidar measurements from the Crop Wind Energy eXperiment (CWEX) in Iowa ${ }^{5}$ and the Scaled Wind Farm Technology (SWiFT) facility in Texas. It has also been shown in large-eddy simulations by Xie and Archer, ${ }^{6}$ Bhaganagar and Debnath, ${ }^{7}$ and Lundquist et al. ${ }^{8}$

The focus of this work is to use high-fidelity flow simulation to examine the effects and importance of wake skew caused by wind veer. Specifically, we are interested in exploring 1) whether or not wake skewing affects

\footnotetext{
*Senior Engineer, National Wind Technology Center, matt.churchfield@nrel.gov, AIAA Member.

${ }^{\dagger}$ Principal Engineer, National Wind Technology Center
} 
wake recovery, and 2) how wake skewing affects the power-generation efficiency and mechanical loading of a waked downstream turbine. Answering these questions will help establish the significance of wake skewing caused by wind veer, which will ultimately provide guidance on how important it is to model the wake-skewing effect in engineering wake models. To our knowledge, the only proposed wake-skewing model is that of Gebraad et al., ${ }^{9}$ which was applied to the FLOw Redirection and Induction in Steady state (FLORIS) engineering wake model. ${ }^{10}$

\section{Method}

To study the effect of wind veer caused by the Earth's Coriolis forces on wind turbine wakes, we use the highest fidelity form of computational fluid dynamics possible for this type of flow, which is large-eddy simulation (LES). With LES, the larger, energy-containing scales of turbulence are directly resolved, but the effect of the smaller ones are modeled using a subgrid-scale turbulence model. The flow solver is the National Renewable Energy Laboratory's (NREL's) Simulator fOr Wind Farm Applications (SOWFA) tool, ${ }^{11}$ which is a custom set of wind-power-plant-specific libraries built on top of the well-known and widely used OpenFOAM computational fluid dynamics tool kit. ${ }^{12}$ The filtered incompressible Navier-Stokes equations are solved in the cell-centered, collocated-variable, unstructured, finite-volume formulation. Density stratification effects are accounted for by including a Boussinesq buoyancy term. The Coriolis term is included in the momentum equations, which is essential to this study. The subgrid-scale turbulence model is the Deardorff one-equation model shown by Moeng. ${ }^{13}$ We perform wall-modeled LES using Schumann's ${ }^{14}$ rough wall surface stress model at the ground boundary. The turbine rotor aerodynamics are represented with actuator lines, which is a technique in which each blade is modeled as a rotating, deformable line of body force applied to the flow field. The body force distribution along each line comes from the lift and drag distribution along the blade. Our implementation of the rotor actuator line method follows that of Sørensen and Shen ${ }^{15}$ with some refinements outlined by Churchfield et al. ${ }^{16}$ An actuator approach is also used to model the drag of the tower and nacelle. ${ }^{17}$ The actuator line is coupled with NREL's FAST aeroelastic and system dynamic tool. ${ }^{18}$ The flow solver passes velocity information along the actuator lines to FAST, and FAST returns forces and blade, tower, and nacelle positions. In this way, the turbine structural motion, along with the control system's effect on rotor speed and position, is captured within the flow solver.

The simulation process is performed in two steps. First, we perform two precursor atmospheric large-eddy simulations, one with Coriolis forces and one without. From this point forward, we will refer to the Coriolis and non-Coriolis cases as the "veer" and "no-veer" cases. In these simulations, the lateral boundaries are periodic, and the ground boundary is flat. In these cases, we use a domain that is $3000 \mathrm{~m} \times 2000 \mathrm{~m}$ in the horizontal and $800 \mathrm{~m}$ in the vertical with a uniform $5-\mathrm{m}$ resolution. The simulations are initialized to a uniform flow speed with organized perturbations near the surface. The temperature is initialized to $300 \mathrm{~K}$ with a capping inversion of $0.003 \mathrm{~K} / \mathrm{m}$ beginning $500 \mathrm{~m}$ above the surface. To form significant wind veer because of Coriolis forces, a low turbulence level is required to reduce vertical mixing of momentum. Therefore, we set a surface cooling rate of $0.125 \mathrm{~K} / \mathrm{hr}$. For the case that included Coriolis forces, the rotation period was set to the 24 -hr period of the Earth at a north latitude of $57.5^{\circ}$. In this case, the flow is driven to a mean speed of $8 \mathrm{~m} / \mathrm{s}$ at the turbine hub height with the winds from the west. This is achieved by sampling the flow in the hub-height plane and spatially averaging it. The planar-averaged value is compared with the desired mean speed, and then a correction to a spatially uniform momentum source term is applied that is proportional to the wind speed error multiplied by the time step. This process is repeated each time step and can be thought of as a controller with gain proportional to the wind speed error. After a few thousand seconds, the momentum source fluctuates about a steady value, and the fluctuation amplitude is small. We allow each simulation to run until the atmospheric boundary layer reaches a quasi-equilibrium state at $16,000 \mathrm{~s}$. This is defined as the time at which the planar-averaged wind speed at different heights passes an initial transient, and the turbulent stress profiles become fully developed. At that point, we save an additional $3600 \mathrm{~s}$ of time history of velocity and temperature over what will become the inflow boundary of the second simulation set (the inflow/outflow cases that include wakes) in addition to the spatially constant source data that maintains a given mean flow speed at a certain height.

Next, we run the wake case. The same overall domain is used, and it is initialized to the state of the precursor at $16,000 \mathrm{~s}$. The domain is refined around the turbine and its wake to a resolution of $1.25 \mathrm{~m}$. This is done by successively selecting sets of cells and cutting them in half in each direction. Instead of using periodic boundary conditions on the east and west sides of the domain, we now use inflow and outflow 
conditions. On the west side, the inflow Dirichlet condition is to use the saved time history of velocity and temperature data on that plane from the precursor case. On the west side, the outflow Neumann condition is that velocity and temperature have a zero boundary-normal gradient, unless the flow is trying to come back into the domain, in which case it reverts to a Dirichlet condition of some user-specified value. The actuator line/FAST-modeled turbine is a realistic, large, modern offshore multimegawatt turbine.

While this second wake case set is running, vertical planes of wake data situated normal to the flow are sampled at high frequency from -2 to +10 rotor diameters $(D)$ downstream of the rotor. The data contained in those planes will be used for two purposes: 1) to examine wake behavior and 2) to use as input to FAST runs to examine loads caused by wakes with and without wind veer skewing of the wake.

\section{Results}

\section{III.A. Atmospheric Inflow}

Figure 1 shows the mean vertical structure of inflow winds for the veer and no-veer cases. Both cases have the same mean hub-height wind speed and direction, but with the veer case, we see a roughly $8^{\circ}$ linear wind-direction change across the vertical extent of the rotor disk. By including the Coriolis forces in the veer case, not only is the cross-stream component of the flow modified, but we also see differences in the streamwise component of the flow. In the veer case, the wind speed at the upper and lower reaches of the rotor plane (as denoted by the black dashed lines), are reduced and increased, respectively, relative to the no-veer case, effectively reducing the vertical wind shear slightly.
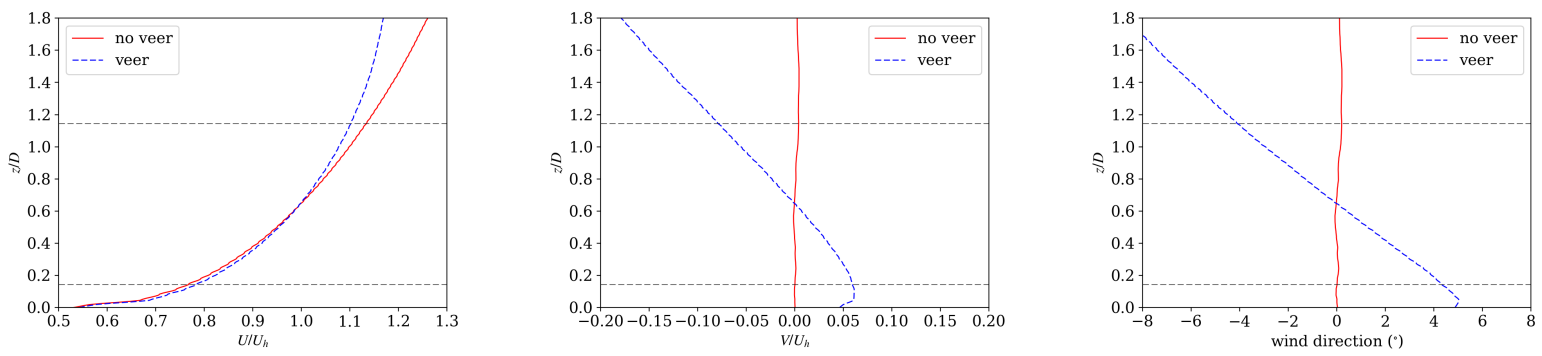

Figure 1. Vertical profile of the mean inflow wind from the veer and no-veer cases: (a) streamwise velocity, (b) cross-stream velocity, and (c) wind direction.

\section{III.B. Wake Flow}

Combining the inflow characterized in Fig. 1 with the actuator line rotor model, the rotor and wake flow is simulated. Overview images of the rotor and wake flow for the veer case are shown in Figs. 2 and 3. Figure 2 shows isosurfaces of instantaneous vorticity magnitude, which are colored by contours of instantaneous streamwise velocity. The isosurface level was chosen such that only vortical structures in the wake are visible; the atmospheric inflow turbulence structures are usually weaker than those in the wake. One can clearly see the tip and root vortices spiraling downstream about a rotor diameter before they begin to break down. The effect of vertical shear in the inflow wind is visible in that the tip vortices on the upper side of the wake advect downstream faster than those on the lower wake edge and are therefore less closely spaced. The axial velocity is large on the outboard side of the vortices relative to the inboard side because of the vortex rotation direction and the resultant axial flow speed induced by these vortices. Strong vorticity caused by the shear in the wake edge is visible wrapping around the tip vortices, promoting tip-vortex instability and eventual breakdown. Even in this relatively low-turbulence stably stratified atmospheric inflow, some wake meandering is evident.

Figure 3 show the planes in and upstream of the wake in which flow sampling is performed. The contours are of instantaneous streamwise velocity. The three components of instantaneous velocity are sampled in these planes at high frequency and saved for later use in running aeroelastic calculations. In the plane directly downstream of the wind turbine, the wake deficit is clearly visible as a dark region in the contour plane. The center of the wake has a high-speed region because the inner portion of the blades create little or no lift and the nacelle's drag has a small effect on the flow relative to the mid to outer blade sections. 


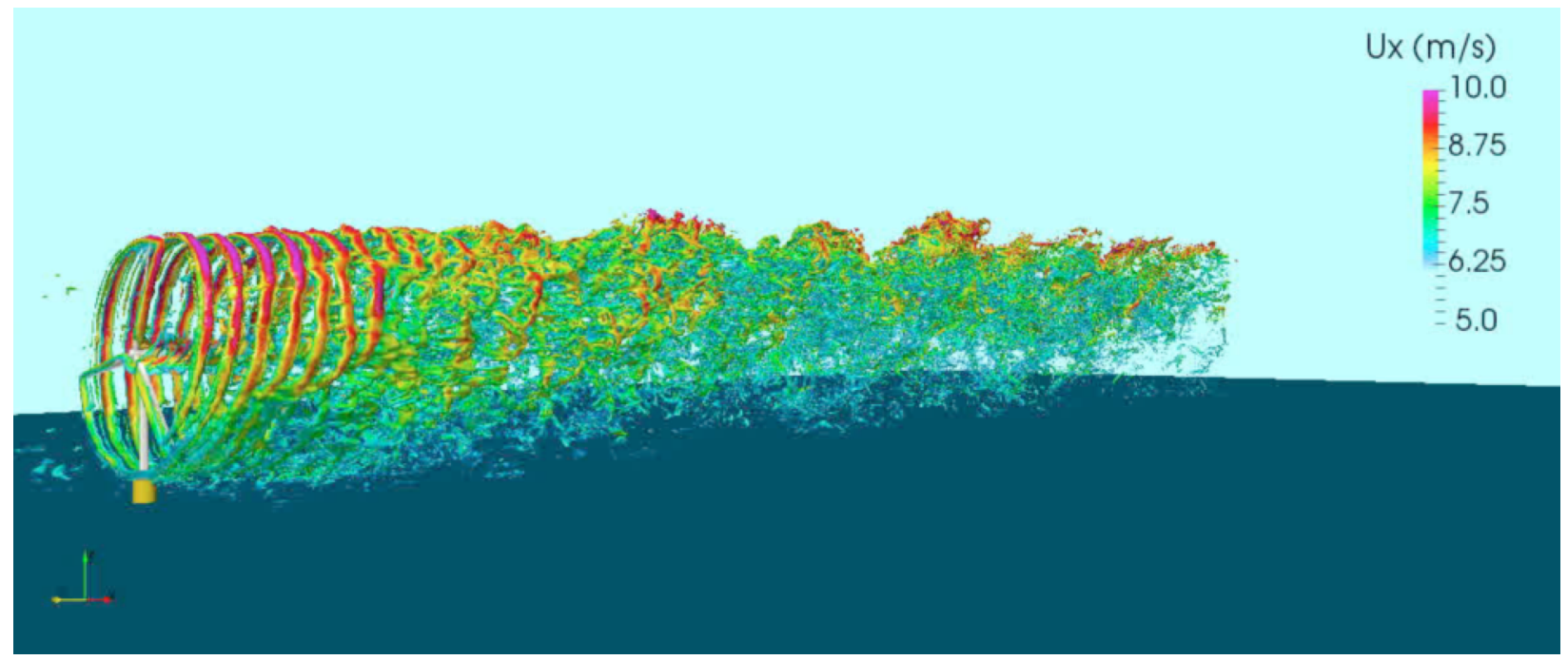

Figure 2. A visualization of the computed wake flow from the case with inflow veer. The isosurfaces are of instantaneous vorticity magnitude and are colored by contours of instantaneous axial velocity.

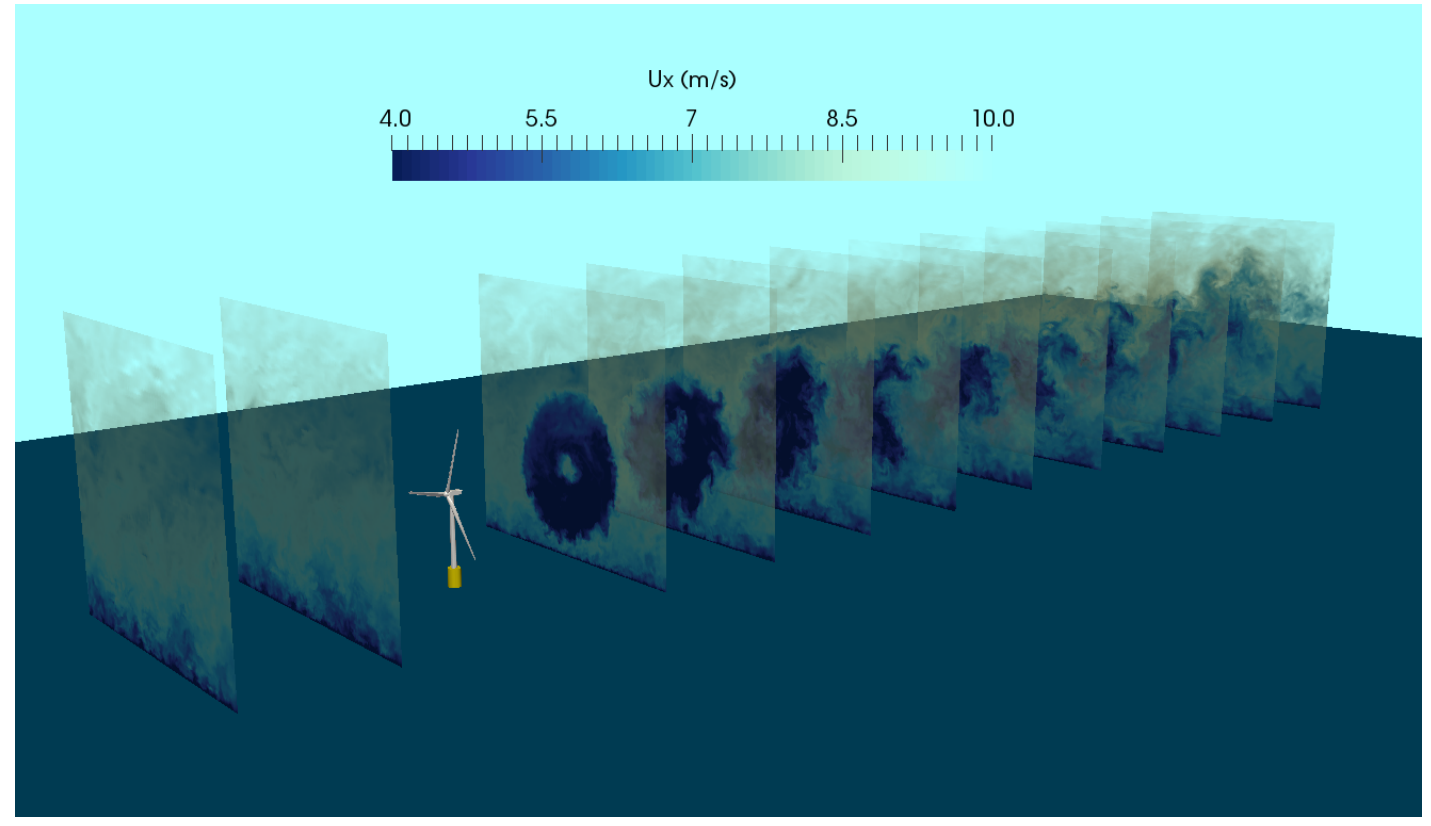

Figure 3. A visualization of the wake flow sampling planes located upstream and downstream of the turbine spaced $1 D$ apart. The contours in the planes are of instantaneous streamwise velocity.

To understand the differences in wake structure with and without veer, it is useful to look at contours of instantaneous streamwise velocity in vertical planes cutting through the wake at different downstream locations, as shown in Fig. 4. In this figure, the no-veer case is on the left and the veer case is on the right. The rotor sweep is projected onto each plane as a red circle. The effect of veer is clearly visible as a skewing of the wake velocity deficit. The upper part of the veered wake is advected in the $-y$-direction and the lower part is advected in the $+y$-direction by the cross-stream component of the inflow. It is also clear that wakes are very unsteady flow features. They contain turbulence generated by the wake edge shear layer, and they interact and mix with turbulence in the atmospheric boundary layer. For this reason, it is important to examine the wake in a more statistical sense.

The time-averaged wake velocity is a very useful statistical quantity to examine first. Figure 5 shows contours of the time-averaged wake in vertical planes at different downstream locations for the veer and noveer cases. The time averaging period is $1600 \mathrm{~s}$. The normalized wake deficit, defined as $1-\frac{\left\langle U_{\infty}(z)\right\rangle-\langle U(y, z)\rangle}{\left\langle U_{h u b}\right\rangle}$, 
is shown in the left two columns. Here, the angle brackets denote time-averaging, $U_{\infty}(z)$ is the streamwise component of the upstream inflow profile, $U(y, z)$ is the streamwise velocity in the wake planes, $z$ is the vertical direction, $y$ is the cross-stream direction, and $U_{h u b}$ is the mean rotor hub-height wind speed. The most notable difference between the wake deficits for each case is the wake skewing because of wind veer. In the no-veer case, the wake is not axisymmetric, especially near the rotor, but it is much more axisymmetric than in the veer case in which the deficit contours are somewhat ellipsoidal and tilted. By $5 D$ downstream, the region of high velocity in the wake center is well mixed and no longer distinguishable. Interestingly, near the turbine there is an acceleration in the flow below the wake, denoted by contour levels greater than one.

In the right two columns of Fig. 5, we show the deviation of the mean cross-stream wake flow from the inflow profile, normalized by the hub-height mean wind speed, which is defined as $\frac{\langle V(y, z)\rangle-\left\langle V_{\infty}(z)\right\rangle}{\left\langle U_{h u b}\right\rangle}$. Here, $V(y, z)$ is the cross-stream wake flow and $V_{\infty}(z)$ is the inflow cross-stream flow profile. Near the rotor, we see distinct positive and negative regions of cross-stream flow deviation, which indicates the rotation of the wake that is the flow's reaction to the rotor torque. In the no-veer case, this cross-stream deviation gradually decays with downstream distance. However, in the veer case, although the cross-stream flow deviation also decays, a significant region of positive cross-stream deviation remains near the top of the wake, whereas a region of negative cross-stream deviation remains in the lower part of the wake. The reason for this is not clear.

One of the questions we wish to answer in this study is whether or not veer affects wake recovery rate. To quantify this, for each downstream sample plane of normalized time-averaged velocity deficit, we extracted the minimum value (value of strongest deficit), and in Fig. 6, we plot this minimum value as a function of downstream distance. It is important to note that the location of this minimum value may change as a function of downstream distance, so this analysis is different than sampling the wake at a constant $y$ and $z$ location as a function of $x$. The wake deficit from the veer case does not attain as low a minimum value as the no-veer case. By $10 D$ downstream, the veer case wake recovers to a value of about 0.72 , whereas the no-veer case has only recovered to about 0.65 . It is not clear why the wake in the veer case recovers more quickly than in the veer case, but one possible explanation is that the skewed wake of the veer case is thinner along its minor axis (if its cross section is thought of as elliptical) than in the no-veer case. Because of this thinner wake, turbulent mixing has less distance over which to transport high momentum fluid to the core of the wake. It would be interesting in future studies to see if wake recovery is even faster if the wind veer angle across the rotor disk is greater than the $8^{\circ}$ of this study.

Another question about wakes subject to veer is whether or not the streamwise momentum deficit skews as would a passive tracer in the veered inflow. Answering this question is important for properly accounting for wake skew in simplified wake models that attempt to incorporate wind veer effects. Further motivation for examining this question comes from the recent work of Bodini, Zardi, and Lundquist ${ }^{5}$ who analyzed field data from a scanning lidar placed within a wind farm to scan wakes. They observed that for a given wind direction difference across the rotor disk vertical extent, the wake skews significantly less than if it acted as a passive tracer in the veered flow.

To answer this question, we use the mean inflow wind profiles to "advect" a set of points that are arranged in a vertical line at the rotor disk downstream to the different planes in which we sample the mean wake flow, as shown in Fig. 7 in white. The advection time is height dependent because the flow nearer the ground is slower than that further aloft, and we want the points to fully reach each downstream station before measuring the skew angle. Because the inflow wind veer profile is not perfectly linear, the originally linear set of points (solid white) does not remain linearly arranged (dashed white), so we fit a line to the points (solid magenta). We then measure the angle of the line with respect to vertical at each downstream location. We will call this angle the "expected" skew angle. Next, in each downstream sample plane, we scan across the plane horizontally at many different vertical levels to find the minima in the wake deficit profile at each height (cyan dots). Again, we fit a line to these minima (cyan line) and measure the angle with respect to vertical and call this the "actual" skew angle. This process for quantifying wake skew angle assumes that with no veer, the wake deficit minima points would be arranged in a nearly vertical line, which we found to be true as shown Fig. 7(a). We also "advected" a set of points originally on the rotor disk circle (solid red) to see how their shape visually looks when skewed (dashed red) compared to the wake shape.

In Fig. 8, we plot the ratio of actual-to-expected wake skew angle. From $2 D$ downstream and beyond, the ratio is less than one meaning that the wake does not skew as much as if it were a passive tracer. This ratio generally decreases with downstream distance, and by $10 D$, the ratio is about 0.8 . This value of 0.8 agrees with some of Bodini, Zardi, and Lundquist's ${ }^{5}$ observed data for similar wind veer angles, especially 
for wakes from turbines on the outer edges of the wind farm that do not experience wake interactions. The isolated turbine simulated in this study would be most similar to the turbines on the outer edges of the wind farm.

The reason that the actual-to-expected wake skew angle ratio is less than one is complex. First, the wake deficit is not a passive tracer, but rather is advected by the wake itself. The wake modifies the cross-stream flow, which was pointed out in the discussion regarding the mean cross-stream flow deviation shown in the right side of Fig. 5. In Fig. 5, we noticed that the upper part of the wake has a positive cross-stream deviation and the lower part of the wake has a negative deviation. This cross-stream deviation pattern persists all the way to $10 D$ downstream, and the direction of the deviations would counteract the skewing effect of the cross-stream component of the inflow. That counteraction would make the actual-to-expected skew angle less than one.
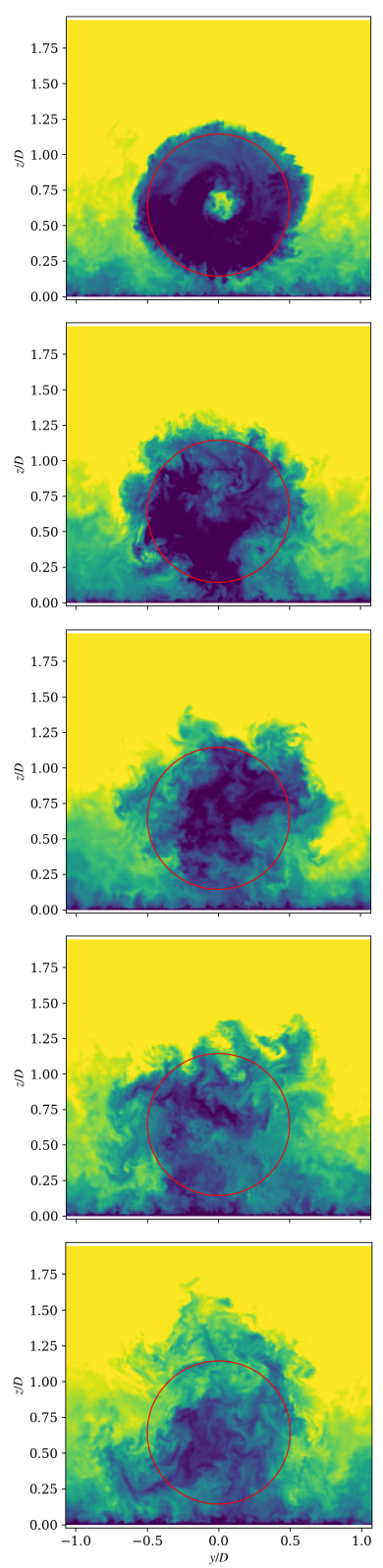

no veer
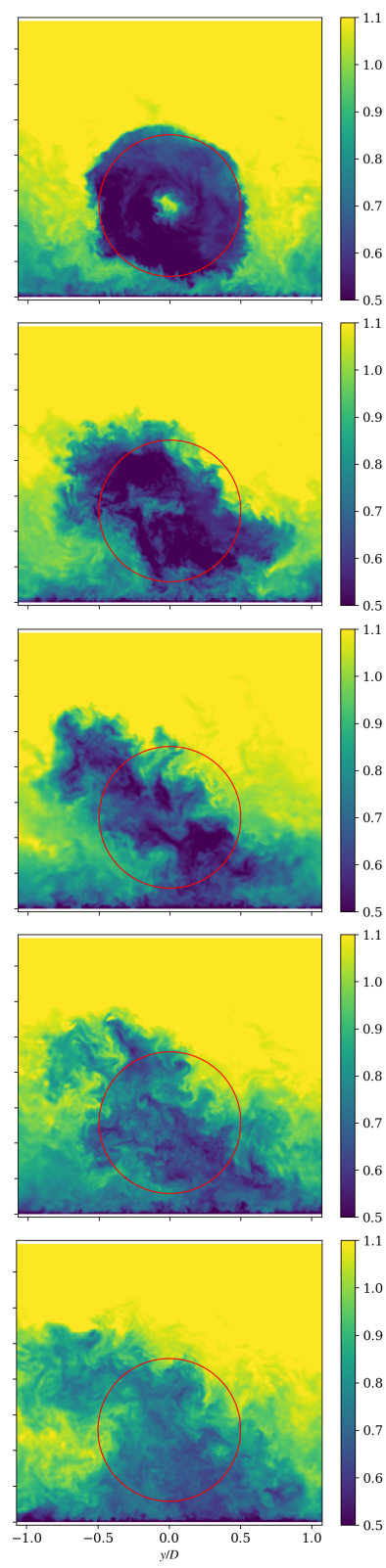

veer

Figure 4. Contours of instantaneous axial velocity normalized by the mean hub-height wind speed $\left(U(y, z) /\left\langle U_{h u b}\right\rangle\right)$ in transverse vertical planes at different downstream locations for the no-veer (left) and veer (right) cases. The downstream locations shown from top to bottom are $1 D, 3 D, 5 D, 7 D$, and $9 D$. The flow is viewed from downstream looking toward upstream. The red circle is the rotor circle projected downstream onto each plane. 

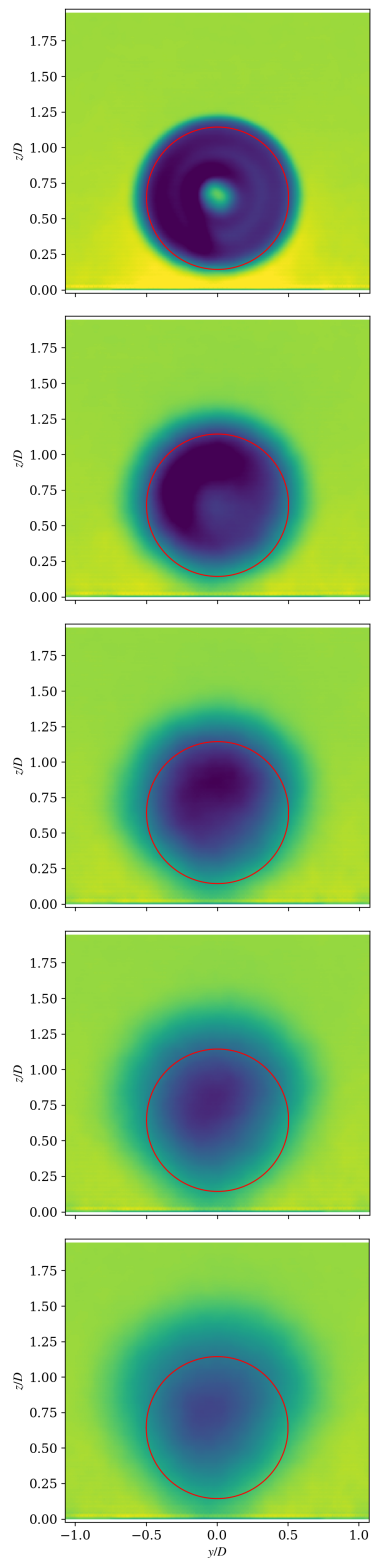

no veer
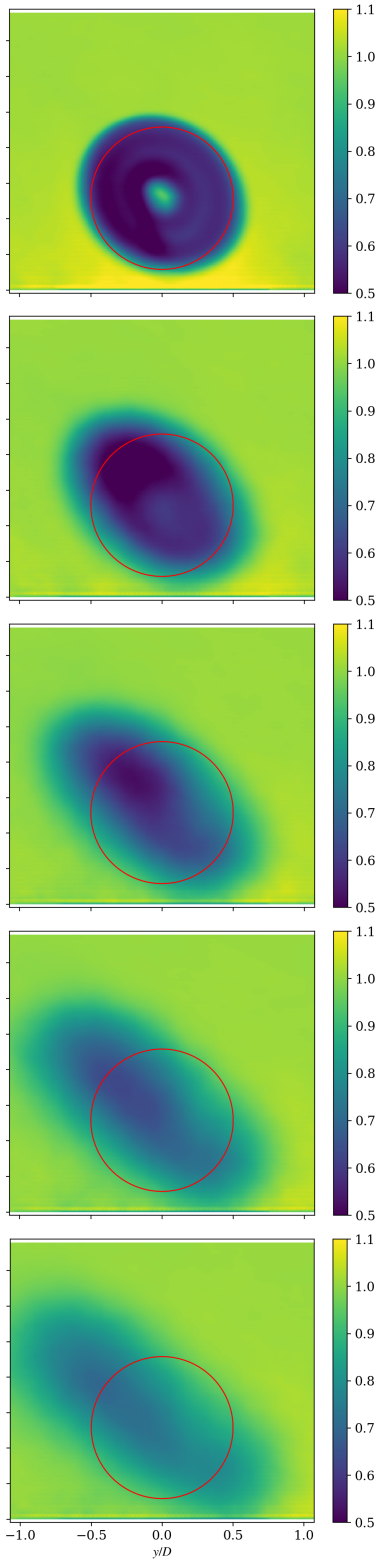

veer

$$
1-\frac{\left\langle U_{\infty}(z)\right\rangle-\langle U(y, z)\rangle}{\left\langle U_{h u b}\right\rangle}
$$
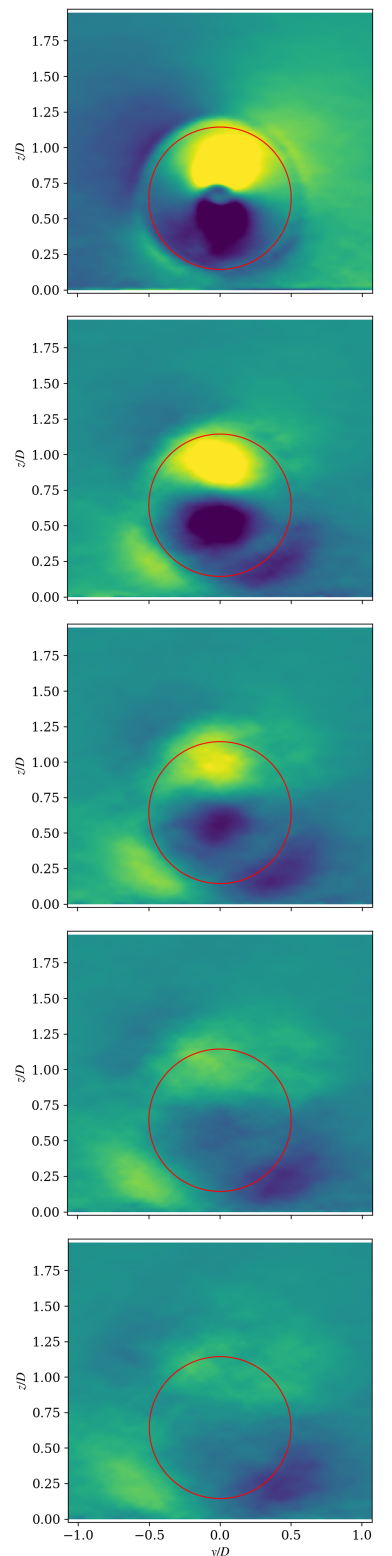

no veer
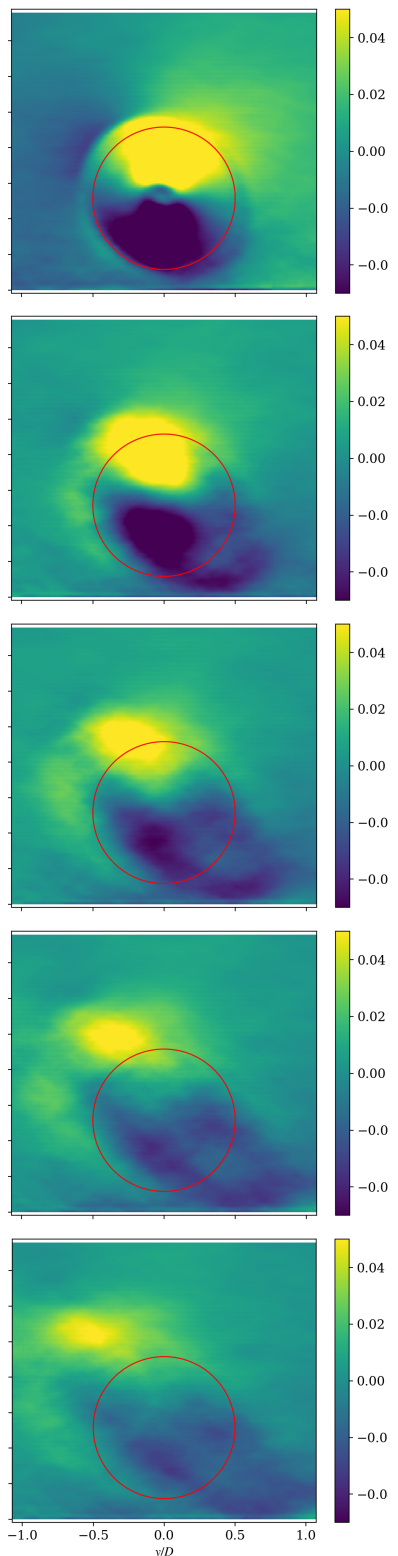

veer

$$
\frac{\langle V(y, z)\rangle-\left\langle V_{\infty}(z)\right\rangle}{\left\langle U_{h u b}\right\rangle}
$$

Figure 5. Contours of time-averaged axial velocity deficit normalized by the mean hub-height wind speed $\left(1-\left(\left\langle U_{\infty}(z)\right\rangle-\langle U(y, z)\rangle\right) /\left\langle U_{h u b}\right\rangle\right)$ (left two columns) and time-averaged cross-stream velocity deviation normalized by the mean hub-height wind speed $\left(\left(\langle V(y, z)\rangle-\left\langle V_{\infty}(z)\right\rangle\right) /\left\langle U_{h u b}\right\rangle\right)$ (right two columns)in transverse vertical planes at different downstream locations. For each column pair, the no-veer case is to the left and veer case is to the right. The downstream locations shown from top to bottom are $1 D, 3 D, 5 D, 7 D$, and $9 D$. The flow is viewed from downstream looking toward upstream. The red circle is the rotor circle projected downstream onto each plane. 


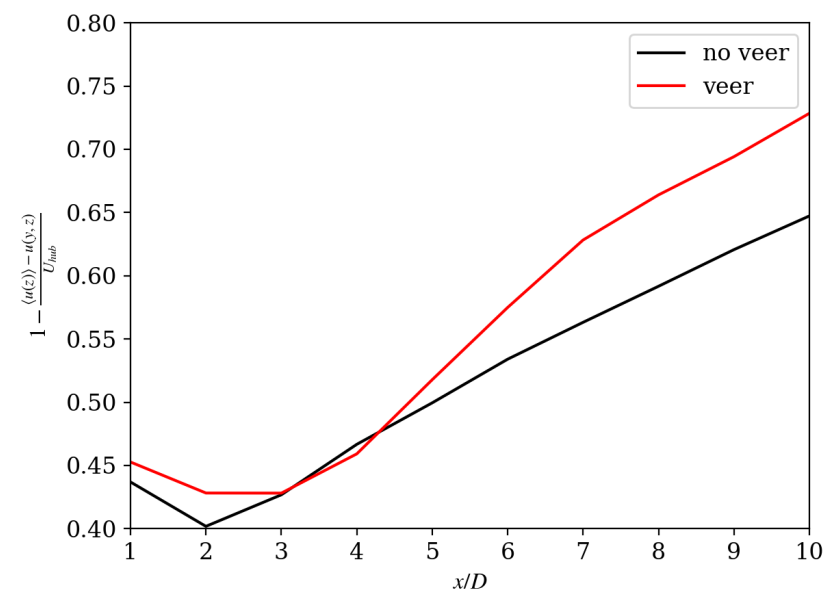

Figure 6. Maximum time-averaged axial velocity deficit normalized by the mean hub-height wind speed as a function of downstream distance, $x$. A value of one indicates no velocity deficit; a value of zero indicates no flow.

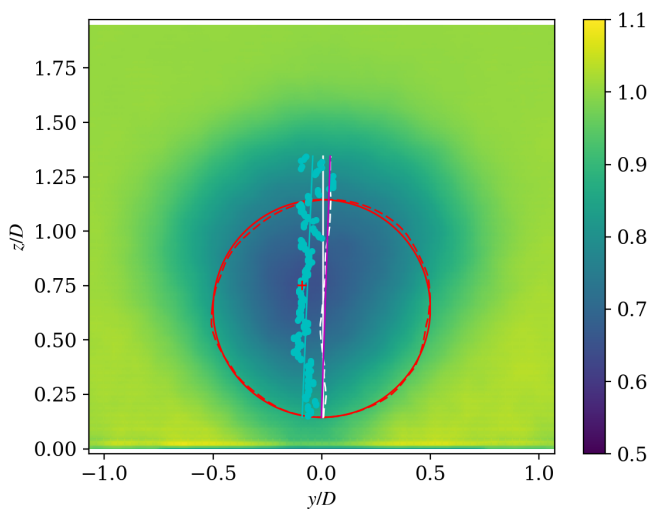

(a)

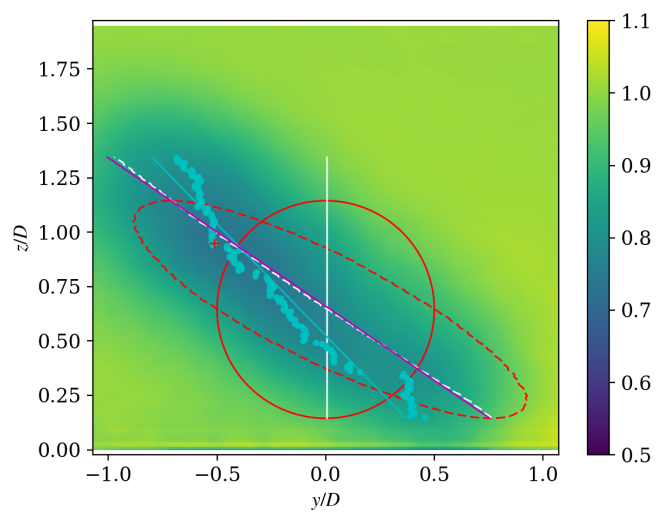

(b)

Figure 7. The normalized mean streamwise wake deficit in a vertical plane at $10 D$ downstream from the no-veer (a) and veer (b) case. The solid white line and solid red circle denotes the original location of the "advected" line and rotor circle, respectively. The dashed white line and dashed red circle denote the skewed "advected" line and rotor circle, respectively. The magenta line is the best fit to the advected line. The cyan dots are the locations of the wake deficit minima in horizontal lines traversing this sample plane, and the cyan line is the best fit to the cyan points.

\section{III.C. Power and Mechanical Loads}

The purpose of using an engineering wake model is to predict power production and mechanical loading under waked conditions. Although the fluid mechanics of wake skewing caused by inflow veer is very interesting, ultimately we want to know if wake skew has an appreciable impact on power and loads to determine how important a feature this is to include in future engineering wake models. To examine the impact on power and loads, we used the wake velocity field data from $9 D$ downstream as input to aeroelastic simulations using NREL's FAST ${ }^{18}$ aeroelastic tool with the same multimegawatt turbine model used to generate the wakes with LES. We place this downstream turbine at three different lateral positions: $y=-0.5 D$, $0 D$, and $+0.5 D$ to simulate direct waking and partial waking. Wake data from both the no-veer and veer cases were used such that we performed six total aeroelastic simulations. Figure 9 shows the normalized mean streamwise velocity in the wake at $9 D$ downstream for the no-veer and veer case, and the rotor lateral positions for each set of aeroelastic simulations are shown as circles. It is important to emphasize that these simulations were not aeroelastic simulations embedded within the LES solver, as was done to generate the wakes, but rather stand-alone aeroelastic simulations using the already generated wake data analyzed earlier.

First, we examine the time-averaged power produced by the downstream turbine in the different lateral 


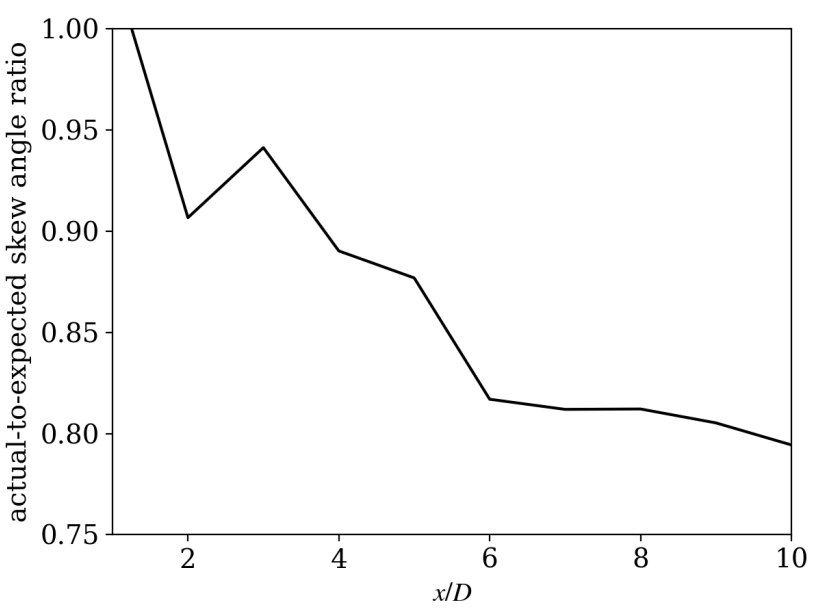

Figure 8. The ratio of actual-to-expected wake skew angle from the veer case as a function of downstream distance.

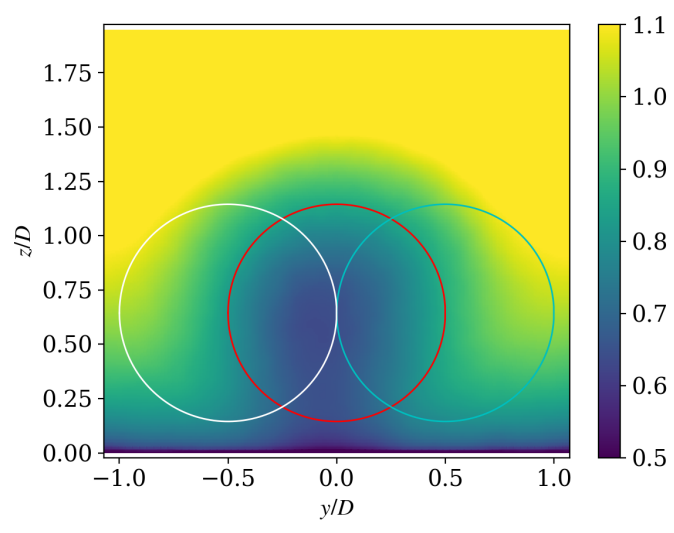

(a)

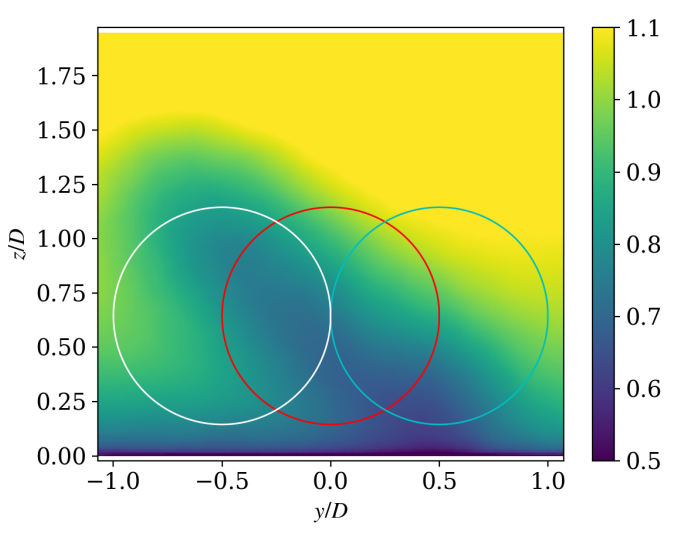

(b)

Figure 9. The normalized mean streamwise velocity $\left(\langle U\rangle /\left\langle U_{h u b}\right\rangle\right)$ in a vertical plane at $9 D$ downstream from the no-veer (a) and veer (b) case. The circles show the lateral placement of the turbines for the aeroelastic simulations: $y=-0.5 D$ (white), $0 D$ (red), and $+0.5 D$ (cyan).

positions and subject to the skewed versus nonskewed wake. The mean power normalized by the no-veer, no lateral offset case is shown in Fig. 10. We see that for both the cases subject to the skewed and nonskewed wakes, a lateral offset in the turbine causes the turbine to produce greater power than in the nonoffset case. We expect that for the nonoffset case the power would be the lowest because the turbine is placed fully within the low-speed wake, but with a lateral offset, the turbine experiences some of the higher-speed undisturbed winds. More interesting for this study, though, we see that for the $0 D$ and $+0.5 D$ offset positions, the turbine subject to the skewed wake produces more power than the turbine subject to the nonskewed wake. With no lateral offset, the turbine in the skewed wake case produces $30 \%$ more power than the turbine in the nonskewed wake case. For the $-0.5 D$ case, the turbine subject to the skewed wake produces less power than the turbine subject to the nonskewed wake. By examining the mean streamwise flow at $9 D$ relative to the rotor positions shown in Fig. 9, this power behavior can be better understood. For the skewed wake case, we can observe that for a $+0.5 D$ lateral offset, the rotor disk contains more high-speed undisturbed flow than the $-0.5 D$ lateral offset rotor disk, hence, it produces more power. We also saw in Fig. 6 that the deficit of the skewed wake is about $15 \%$ more recovered by $9 D$ than that of the nonskewed wake, which explains why for no lateral offset, the turbine subject to the skewed wake produces significantly more power than that subject to the nonskewed wake.

Next, we examine some of the mechanical loading. We have chosen to examine blade-root out-of-plane 


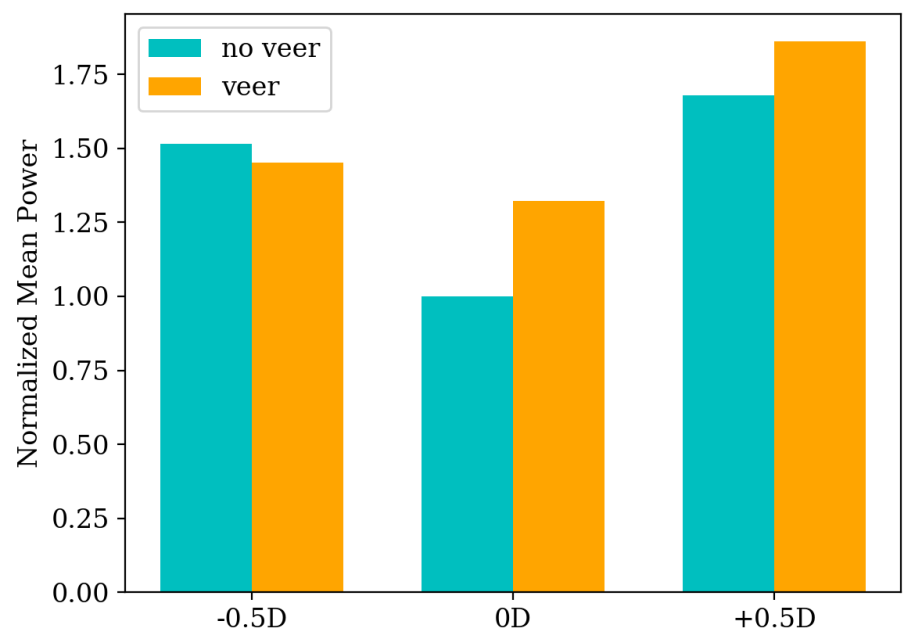

Figure 10. Time-averaged power produced by a turbine subject to the no-veer (cyan) and veer (orange) wake of a turbine $9 D$ upstream. The downstream turbine is placed at $y=0.5 D, 0 D$, and $-0.5 D$ laterally. The power is normalized by power from the no-offset, no-veer case.

(OOP) bending moment, tower-top yaw moment, and tower-top pitch moment. The mean values and damage-equivalent loads (DELs) are shown in Fig. 11. In all cases, we normalize the mean and DEL values by the no-veer, no-lateral-offset case.

The DEL is defined as the load range that causes the same lifetime damage as the actual computed load cycles. It is assumed to have a zero mean load range to be applied for 20 years at a frequency of $1 \mathrm{~Hz}$. To determine DELs, fatigue analysis was conducted using MLife, ${ }^{19}$ a program that uses techniques specified by International Electrotechnical Commission 61400-1, Ed. 3, Annex G. ${ }^{20}$ MLife computes short-term DELs from the available time-series output by each FAST simulation and integrates these DELs over the design lifetime to estimate lifetime DELs based on the Weibull wind distribution. The number of cycles determines the consequential damage from a given set of time history data.

The mean blade-root OOP bending moment follows the trends seen with power. This is expected because in below-rated operation, as the rotor is subject to higher inflow wind speeds to produce more power, there is more rotor thrust, which also increases the mean blade-root OOP bending. The blade-root OOP bending moment DELs are larger for cases with lateral offset than without because the rotor is now partially waked, meaning that the blade passes in and out of the wake every time it completes a revolution. This cyclic passage from high-speed to low-speed flow creates larger amplitude blade-root OOP bending moment fluctuations, which increases fatigue and DEL. For the $y=0 D$ and $+0.5 D$ cases, the rotor subject to the skewed wake has higher blade-root OOP bending DELs than for the rotor subject to the nonskewed wake. For the $y=-0.5 D$ case, though, the situation is opposite. The best way to understand this it to examine Fig. 9. The degree of asymmetry of the inflow velocity across the rotor disk positively correlates with the level of the DEL.

The mean tower-top yaw moment is an interesting quantity in that it is a result of lateral asymmetry in rotor loading. Rotor loading is directly related to the inflow velocity, so cases that have lateral asymmetry in inflow velocity exhibit a finite tower-top yaw moment. The cases with rotor lateral offset are subject to the partial waking situation in which one side of the rotor is waked and the other is unwaked, which causes lateral loading asymmetry and mean positive or negative tower-top yaw moment. The sign of the mean quantities is misleading because they seem opposite of what they should be, but that is true because the normalization case is negative. Moving to the tower-top yaw moment DELs, we see that the DELs for the rotor subject to the skewed wake are greater than those of the rotor subject to the nonskewed wake, regardless of lateral position. The reason for this is unclear but may point to differences in the unsteady behavior of the skewed versus nonskewed wakes. For example, if they meander differently, the DELs would then be different.

The mean tower-top pitch moment is similar to tower-top yaw moment in that it is caused by rotor loading asymmetry, but this time by vertical asymmetry. Again it is helpful to refer to Fig. 9 to understand 
the flow asymmetry. The one anomaly is for the case in which the rotor is subject to the skewed wake and offset to $y=+0.5 D$. Here, the mean tower-top pitch moment is much smaller in magnitude and opposite in sign of all the other cases. When we examine the mean flow for this case in Fig. 9, we see the strongest vertical asymmetry in the flow of any case, with much higher velocity near the top of the rotor. It may be because without any aerodynamic loading, gravity loads create a negative mean pitching moment, but in this case, the flow asymmetry was just strong enough to cause the aerodynamic moment to counteract the gravity moment. Like with the mean tower-top yaw moment, the signs of the normalized quantities are misleading because the normalization case has a negative value. For this moment, there are differences in the DELs for the rotor subject to the skewed versus nonskewed wake, but the differences are smaller and seem more random than for other loads quantities.

\section{Conclusion}

In this work, we performed LES of atmospheric inflow with and without Coriolis forces caused by the Earth's rotation. In the case with Coriolis forces included, wind direction veer with height develops. The veer can be significant during stably stratified conditions. We performed a slightly stably stratified simulation resulting in a wind-direction change across the vertical extent of a large, modern, multimegawatt turbine rotor of about $8^{\circ}$. We then used these inflow data to drive a second set of simulations that include an actuator line rotor aerodynamics model for this large multimegawatt turbine, which produces wakes. We sampled the instantaneous wake flow every $1 D$ downstream to $10 D$ downstream at a high frequency and 1.25 $\mathrm{m}$ resolution. We then used those sampled downstream wake data to compare and contrast wakes subject to veered and nonveered flow. The wake in the veered inflow case clearly skews as it advects downstream. We also used the sampled wake data to drive aeroelastic simulations of a hypothetical downstream turbine subject to the two different wakes to examine the affect on power and mechanical loads.

One key finding is that wake skewing caused by veered inflow is not a simple passive-tracer-like process. The wake clearly skews as one may expect, but its skew angle is less than expected if the wake behaved like a passive tracer. The ratio of the wake skew angle relative to the expected skew angle decreases with downstream distance, but at $10 \mathrm{D}$ downstream, the ratio is roughly $80 \%$. This outcome agrees with the lidar field measurements of Bodini et al. ${ }^{5}$ Also, we found that the skewed wake recovers faster than the nonskewed wake. We hypothesize that because the wake deficit is skewed such that it has a narrowed elliptical transverse cross section, turbulent transport of high-speed nonwake flow to the center of the wake is more efficient because the wake cross section is narrower than in the non-skewed case.

The aeroelastic simulations of the hypothetical downstream turbine were also illuminating. We observed that when this downstream turbine was directly downstream the upstream turbine or laterally offset in the positive $y$-direction, the power generated was higher for the case using the skewed wake. However, the opposite is true if the turbine is offset in the negative $y$-direction. We also see differences in the mechanical loading quantities we examined, which are blade-root out-of-plane bending moment, tower-top yaw moment, and tower-top pitch moment. For the mean tower-top moments and blade-root out-of-plane bending DELs, behavior can be linked back to the mean flow asymmetry across the rotor disk, and wake skew significantly influences this asymmetry.

We conclude that wake skew caused by wind direction veer in the inflow winds is an important feature to capture in engineering wake models. It has impacts on wake recovery and hence power generation of downstream turbines. It also modifies mechanical loads. A simple wake skewing using a passive-tracer process with knowledge that wakes skew less than expected may be a good first step toward adding this feature to engineering wake models. This study is meant to show the general features and effects of wake skewing, but it is not an exhaustive study. A worthwhile next step would be to simulate wake skewing under a wider range of conditions. For example, wake skewing should be studied under different levels of wind veer across the rotor disk and different ambient turbulence levels. 

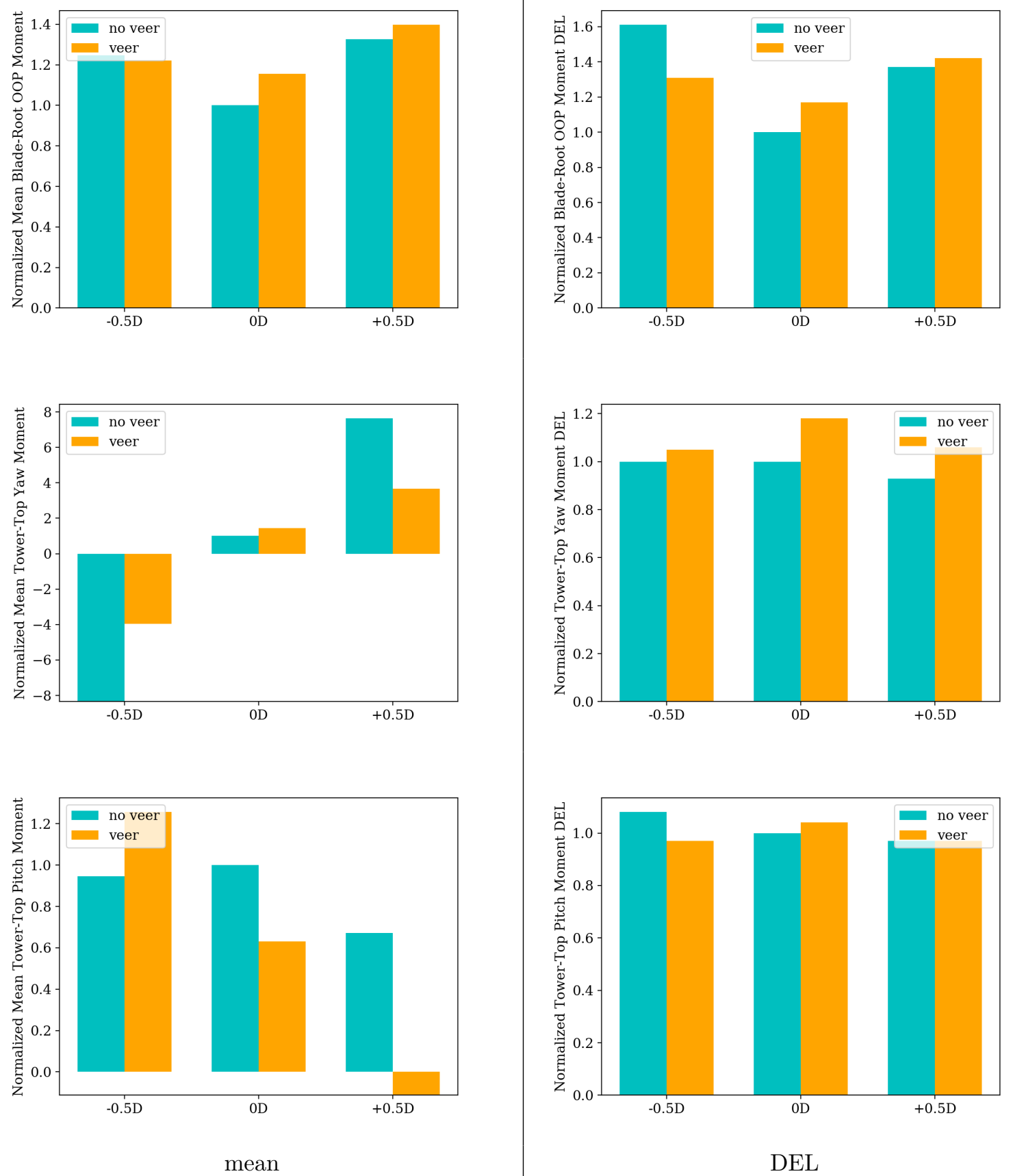

Figure 11. Normalized mean loading quantities (left column) and normalized damage equivalent loads (right column) for blade-root out-of-plane bending moment (top row), tower-top yaw moment (middle row), and tower-top pitch moment (bottom row). 


\section{Acknowledgements}

This work was supported by the U.S. Department of Energy (DOE) under Contract No. DE-AC3608GO28308 with the National Renewable Energy Laboratory. Funding for the work was provided by Statoil ASA. Computations were performed on the National Renewable Energy Laboratory's Peregrine highperformance computing system. The authors thank Andreas Knauer, Marte Godvik, and Bjørn Skaare of Statoil for their collaboration and technical discussion on this work. We acknowledge Finn Gunnar Nielsen of the University of Bergen for proposing some of the initial questions that motivated this work. We also thank Tony Martínez, Eliot Quon, and Paula Doubrawa of NREL for their useful discussion and help with this work.

The U.S. Government retains and the publisher, by accepting the article for publication, acknowledges that the U.S. Government retains a nonexclusive, paid-up, irrevocable, worldwide license to publish or reproduce the published form of this work, or allow others to do so, for U.S. Government purposes.

\section{References}

${ }^{1}$ Jensen, N. O., "A Note on Wind Generator Interaction," Tech. Rep. M-2411, Ris $\varnothing$ National Laboratory, Roskilde, Denmark, 1983.

${ }^{2}$ Katíc, I., Højstrup, J., and Jensen, N., "A Simple Model for Cluster Efficiency," European Wind Energy Association Conference and Exhibition, Rome, Italy, European Wind Energy Association, 1986.

${ }^{3}$ Ainslee, J. F., "Calculating the Flowfield in the Wake of Turbines," Journal of Wind Engineering and Industrial Aerodynamics, Vol. 27, 1985, pp. 213-224.

${ }^{4}$ Larsen, G. C., Madsen, H. A., Bingöl, F., Mann, J., Ott, S., Sørensen, J. N., Okulov, V., Troldborg, N., Nielsen, M., Thomsen, K., Larsen, T. J., and Mikkelsen, R., "Dynamic Wake Meandering Modeling," Tech. Rep. R-1607(EN), Ris $\varnothing$ National Laboratory, Roskilde, Denmark, 2007.

${ }^{5}$ Bodini, N., Zardi, D., and Lundquist, J. K., "Three-Dimensional Structure of Wind Turbine Wakes as Measured by Scanning Lidar," Atmospheric Measurement Techniques, Vol. 10, 2017, pp. 2881-2896.

${ }^{6}$ Xie, S. and Archer, C., "A Numerical Study of Wind-Turbine Wakes for Three Atmospheric Stability Conditions," Boundary-Layer Meteorology, 2017.

${ }^{7}$ Bhaganagar, K. and Debnath, M., "The Effects of Mean Atmospheric Forcings of the Stable Atmospheric Boundary Layer on Wind Turbine Wake," Journal of Renewable and Sustainable Energy, Vol. 7, No. 01324, 2015.

${ }^{8}$ Lundquist, J. K., Churchfield, M. J., Lee, S., and Clifton, A., "Quantifying Error of Lidar and Sodar Doppler Beam Swinging Measurements of Wind Turbine Wakes Using Computational Fluid Dynamics," Atmospheric Measurement Techniques, Vol. 8, 2015, pp. 907-920.

${ }^{9}$ Gebraad, P. M. O., Churchfield, M. J., and Fleming, P. A., "Incorporating Atmospheric Stability Effects into the FLORIS Engineering Model of Wakes in Wind Farms," The Science of Making Wind From Torque, Munich, Germany, Oct. 5-7, 2016, 2016.

${ }^{10}$ Gebraad, P., Teeuwisse, F., Wingerden, J., Fleming, P. A., Ruben, S., Marden, J., and Pao, L., "Wind Plant Power Optimization Through Yaw Control Using a Parametric Model for Wake Effects-A CFD Simulation Study," Wind Energy, Vol. 19, No. 1, 2016, pp. 95-114.

${ }^{11}$ Churchfield, M. and Lee, S., "Simulator for Wind Farm Applications (SOWFA)," available online at https://nwtc.nrel.gov/SOWFA, accessed June 27, 2017, created by National Renewable Energy Laboratory.

${ }^{12}$ OpenCFD Ltd. (ESI Group), "OPENFOAM - The Open Source CFD Toolbox," available online at http://www.openfoam.com, accessed February 26, 2016.

${ }^{13}$ Moeng, C.-H., "A Large-Eddy-Simulation Model for the Study of Planetary Boundary-Layer Turbulence," Journal of the Atmospheric Sciences, Vol. 41, No. 13, 1984, pp. 2052-2062.

${ }^{14}$ Schumann, U., "Subgrid-scale Model for Finite Difference Simulation of Turbulenct Flows in Plane Channels and Annuli," Journal of Computational Physics, Vol. 18, 1975, pp. 376-404.

${ }^{15}$ Sørensen, J. N. and Shen, W. Z., "Numerical Modeling of Wind Turbine Wakes," Journal of Fluids Engineering, Vol. 124, 2002, pp. 393-399.

${ }^{16}$ Churchfield, M. J., Schreck, S. J., Martínez, L. A., Meneveau, C., and Spalart, P. R., "An Advanced Actuator Line Method for Wind Energy Applications and Beyond," AIAA SciTech Forum, Grapevine, Texas, Jan. 9-13, 2017, AIAA, Washington D.C., 2017, AIAA Paper 2017-1998.

${ }^{17}$ Churchfield, M. J., Wang, Z., and Schmitz, S., "Modeling Wind Turbine Tower and Nacelle Effects with an Actuator Line Model," AIAA SciTech Forum, Kissimmee, Florida, Jan. 5-9, 2015, AIAA, Washington D.C., 2015, AIAA Paper $2015-0214$.

${ }^{18}$ Jonkman, J., "FAST," available online at https://nwtc.nrel.gov/FAST, accessed June 27, 2017, created by National Renewable Energy Laboratory.

${ }^{19}$ Hayman, G., "MLife: A Matlab-based Estimator of Fatigue Life," available online at https://nwtc.nrel.gov/MLife, accessed December 12, 2017, created by National Renewable Energy Laboratory.

${ }^{20}$ International Electrotechnical Commission, "IEC 61400-1: Wind Turbine - Part 1: Design Requirements," 2014. 